\title{
Role of Multislice CT in Diagnosis, Staging and Evaluation of Malignant Pleural Mesothelioma
}

\author{
Dina Ahmed Hassan Torky, Hoda Mahmoud Abdelwahab, Fadila Mamdouh Elsayed \\ Department of Radiodiagnosis, Faculty of Medicine (girls), Al-Azhar University, Cairo, Egypt \\ Corresponding author: Dina Ahmed Hassan Torky, Mobile: 01017806339; Email: dina.torky1990@ gmail.com
}

\begin{abstract}
Background: Malignant mesothelioma is a rare but fatal disease that arises from the epithelial lining of the pleura, peritoneum, pericardium and tunica vaginalis. Malignant pleural mesothelioma (MPM) is the most common form, accounting for $80-90 \%$ of malignant mesotheliomas

Aim of the work: was to identify the value of CT in diagnosing malignant pleural mesothelioma and applying the AJCC and the IMIG staging system for MPM. At the time to identify the limitations of CT if any.

Patient and methods: This prospective study included a total of 20 patients with CT findings suggestive of malignant pleural mesothelioma, diagnosed at Radiology Department, Damanhur Oncology Center. All patients had undergone multislice CT chest with intravenous contrast for detection and staging of the tumor. This study was conducted between March 2018 and December 2018. The data collected were tabulated and analyzed statistically.

Results: CT study of the chest for cases of MPM was able to evaluate and diagnose the disease, with most of the important staging items being easily seen on CT yet this study also showed the limitations of CT in the staging MPM since CT alone was not able to prove the involvement of the chest wall, diaphragmatic muscle and trans diaphragmatic extension.
\end{abstract}

Conclusions: Chest CT alone is often sufficient for disease staging and treatment planning. Typical CT findings that suggest MPM include unilateral pleural effusion with nodular irregular pleural thickening which can be discrete or diffuse with or without inter lobar fissure thickening and nodal metastasis.

Keywords: Multislice CT - Malignant Pleural Mesothelioma.

\section{INTRODUCTION}

Malignant mesothelioma is a rare but fatal disease that arises from the epithelial lining of the pleura, peritoneum, pericardium and tunica vaginalis. MPM is the most common form, accounting for $80-90 \%$ of malignant mesotheliomas ${ }^{(\mathbf{1})}$. It is the most common primary malignant pleural neoplasm ${ }^{(2)}$. The majority of cases are associated with prior asbestos exposure ${ }^{(3)}$.

The tumor can invade both visceral and parietal pleura and frequently extends to adjacent structures. MPM is locally aggressive with frequent invasion of the chest wall, mediastinum, and diaphragm ${ }^{(4)}$.

The prognosis is poor, with a median survival time of 12 months after diagnosis. Several factors have been shown to correlate with reduced survival time which are intrathoracic lymph node metastases, distant metastatic disease, and extensive pleural involvement ${ }^{(2)}$.

The primary role of imaging in malignant mesotheliomas lies in preoperative staging and assessment of treatment response, disease recurrence, or metastasis ${ }^{(5)}$. CT with contrast is the most frequently obtained examination owing to its easy accessibility ${ }^{(5)}$.

It is the most frequently used modality in the preoperative assessment of patients being considered for surgical resection ${ }^{(4)}$.

Low-dose CT has a greater sensibility than chest Xray to detect tumor at early and treatable stage in screening population ${ }^{(6)}$.

Key CT findings that suggest MPM include unilateral pleural effusion, nodular pleural thickening and inter lobar fissure thickening ${ }^{(2)}$.
Chest wall invasion manifests as obliteration of fat planes or chest wall nodules. There is also frequent contraction of the affected hemithorax with associated ipsilateral mediastinal shift, narrowed intercostal spaces, and elevation of the ipsilateral hemidiaphragm (4). FDG PET/CT which superimposes functional imaging over the anatomical mapping yields a more accurate presentation of mesothelioma ${ }^{(7)}$.

FDG PET is generally good in differentiating benign lesions from malignant mesothelioma, which helps in detecting recurrence and provides prognosting information (staging, survival, mortality, etc) ${ }^{\mathbf{( 1 )}}$.

It is important to stress that a diagnosis of mesothelioma cannot be made exclusively with imaging studies. Biopsy is absolutely essential for the accurate diagnosis of mesothelioma ${ }^{(2)}$.

The aim of the current study was to identify the value of CT in diagnosing malignant pleural mesothelioma and applying the AJCC and the IMIG staging system for MPM. At the time to identify the limitations of CT if any.

\section{PATIENT AND METHODS}

This prospective study included a total of 20 patients with CT findings suggestive of malignant pleural mesothelioma, diagnosed at Radiology Department, Damanhur Oncology Center.

Approval of the ethical committee of Al-Azhar Faculty of Medicine (girls) and a written informed consent from all the subjects were obtained. This study was conducted between March 2018 and December 2018. 
Patients were referred for CT chest examination from the Medical and Surgical Oncology Clinic, Damanhur Oncology Center, Damanhur Chest Hospital and Alexandria University Hospital.

All patients had undergone multislice CT chest with intravenous contrast for detection and staging of the tumor.

\section{Inclusion criteria}

Patients with CT chest findings suggestive of malignant pleural mesothelioma.

\section{Exclusion criteria}

Patients who had contraindication to radiation exposure as pregnant women or contra indication to contrast media as patient who have hypersensitivity or renal failure.

All patients were subjected to:

A. History taking including: Age, residence, occupational exposure of asbestos and local chest symptoms and symptoms of distant metastasis.

\section{B. Clinical general and chest examination.}

C. Laboratory tests: mostly complete blood picture and bleeding profile.

\section{Plain Chest X-ray in PA view.}

E. Multislice CT with IV noniodinated contrast medium examination with multi-detector row CT scanner which will be performed in the craniocaudally direction as follows:
1) Employment of a contrast medium is mandatory. The CT scanning delay should be also set at 6080 seconds to optimize the maximum pleural tumor uptake.

2) Dose of contrast medium: $100 \mathrm{ml}$ intravenous just before the examination.

3) The field-of- view (FOV) due to the tumor growth through the diaphragmatic pillars had to cover a wide area from the lung apex to L3.

4) Slice thickness: $10 \mathrm{~mm}$ contiguous sections.

5) Radiation factors: kv: 120-140 and mAmp: 200.

6) Window-level: lung window 1200-600, mediastinal window 300-30, and bone window 1500-250.

F. Histopathological assessment was done either by U/S guided or CT guided True-Cut biopsy. U/S guided biopsy is the preferred method if the thickening is seen by U/S. If the thickening wasn't visible or accessible via U/S, CT guided biopsy was performed.

\section{Statistics}

The data collected were tabulated and analyzed by SPSS (Statistical package for the social science software) statistical package version IBM compatible computer.

\section{RESULTS}

This study was carried out on 20 malignant pleural mesothelioma patients. Their ages were ranged between 30 to 82 years.

Age: Table 1 shows the age groups between the studied patients.

Table (1): The age groups of the studied patients.

\begin{tabular}{|c|c|c|c|c|c|c|}
\hline & \multicolumn{5}{|c|}{ Age groups } \\
\hline Count & $\mathbf{3 0}-$ & $\mathbf{4 0 -}$ & $\mathbf{5 0 -}$ & $\mathbf{6 0 -}$ & $\mathbf{7 0 -}$ & $\mathbf{8 0 -}$ \\
\hline $\mathbf{\%}$ & 3 & 2 & 5 & 5 & 4 & 1 \\
\hline
\end{tabular}

- Gender: The study group consisted of 13 males and7 females, Table 2.

Table (2): Gender variation among the MPM patients.

\begin{tabular}{|c|c|c|}
\hline & \multicolumn{2}{|c|}{ Sex } \\
\hline & Male & Female \\
\hline Count & 13 & 7 \\
\hline$\%$ & $65.0 \%$ & $35.0 \%$ \\
\hline
\end{tabular}

- Laterality: Ten (50\%) of the cases showed right sided disease equal to ten (50\%) showed left sided disease, Table 3. 
Table (3): Laterality among the studied patients.

\begin{tabular}{|c|c|c|}
\hline \multirow{2}{*}{ Count } & \multicolumn{2}{|c|}{ Side } \\
\hline$\%$ & 10 & 10 \\
\hline$\%$ & $50.0 \%$ & $50.0 \%$ \\
\hline
\end{tabular}

- History of asbestos exposure, Table 4.

Table (4): History of asbestos exposure among the studied cases

\begin{tabular}{|c|c|c|}
\hline \multirow{2}{*}{ Count } & \multicolumn{2}{|c|}{ History of asbestos exposure } \\
\hline & Positive & Negative \\
\hline$\%$ & 8 & 12 \\
\hline$\%$ & $40.0 \%$ & $60.0 \%$ \\
\hline
\end{tabular}

- Fissural involvement: Lung fissures showed a high percentage of involvement in $13(65.0 \%)$ cases, Table 5.

Table (5): Fissural involvement among the studied cases.

\begin{tabular}{|c|c|c|}
\hline \multirow{2}{*}{} & \multicolumn{2}{|c|}{ Fissural involvement } \\
\hline Count & Positive & Negative \\
\hline$\%$ & 13 & 7 \\
\hline$\%$ & $65.0 \%$ & $35.0 \%$ \\
\hline
\end{tabular}

- Pleural effusion: Most cases are associated with pleural effusion in about 11 cases including one case with hydropneumothorax, Table 6 .

Table (6): Pleural effusion among the studied MPM cases.

\begin{tabular}{|c|c|c|c|}
\hline & \multicolumn{3}{|c|}{ Pleural effusion } \\
\cline { 2 - 4 } & Positive & $\begin{array}{c}\text { Hydro- } \\
\text { pneumothorax }\end{array}$ & Negative \\
\hline Count & 10 & 1 & 9 \\
\hline$\%$ & $50.0 \%$ & $5.0 \%$ & $45.0 \%$ \\
\hline
\end{tabular}

- Circumferential pleural involvement, Table 7.

Table (7): Circumferential distribution among the studied patients.

\begin{tabular}{|c|c|c|c|c|}
\hline & \multicolumn{4}{|c|}{ Pleural affection } \\
\hline $\begin{array}{c}\text { Localizer } \\
\text { costal } \\
\text { mass }\end{array}$ & $\begin{array}{c}\text { Costal and } \\
\text { diaphragmatic } \\
\text { pleura }\end{array}$ & $\begin{array}{c}\text { Costal and } \\
\text { Mediastinal } \\
\text { pleura }\end{array}$ & $\begin{array}{c}\text { All- } \\
\text { circumferential }\end{array}$ \\
\hline Count & 2 & 2 & 3 & 13 \\
\hline$\%$ & $10.0 \%$ & $10.0 \%$ & $15.0 \%$ & $65.0 \%$ \\
\hline
\end{tabular}

- Hemi thorax volume: It is decreased in in about 12 $(60.0 \%)$ cases, Table 8.

Table (8): The hemi thorax volume in MPM patients.

\begin{tabular}{|c|c|c|}
\hline \multirow{2}{*}{ Count } & \multicolumn{2}{|c|}{ Hemi-thorax volume } \\
\hline Not affected & Decreased \\
\hline$\%$ & 8 & 12 \\
\hline$\%$ & $40.0 \%$ & $60.0 \%$ \\
\hline
\end{tabular}

- Lung affection, Table 9.

Table (9): Lung affection in MPM patients.

\begin{tabular}{|c|c|c|c|}
\hline & \multicolumn{3}{|c|}{ Lunge affection } \\
& $\begin{array}{c}\text { Hematognous } \\
\text { spread }\end{array}$ & $\begin{array}{c}\text { Lymphangitis } \\
\text { carcinomatosis }\end{array}$ & $\begin{array}{c}\text { Direct } \\
\text { affection }\end{array}$ \\
\hline Count & 6 & 2 & 12 \\
\hline$\%$ & $30.0 \%$ & $10.0 \%$ & $60.0 \%$ \\
\hline
\end{tabular}

- Chest wall invasion Table 10.

Table (10): Chest wall invasion in the studied MPM patients.

\begin{tabular}{|c|c|c|}
\hline \multirow{2}{*}{} & \multicolumn{2}{|c|}{ Chest wall invasion } \\
\hline Count & yes & no \\
\hline$\%$ & 2 & 18 \\
\hline$\%$ & $10.0 \%$ & $90.0 \%$ \\
\hline
\end{tabular}

- Pattern of mediastinal affection Table 11.

Table (11): Describes pattern of mediastinal affection

\begin{tabular}{|c|c|c|c|c|}
\hline \multicolumn{5}{|c|}{ Mediastinal affection } \\
\hline & $\begin{array}{c}\text { Transmural } \\
\text { pericardial } \\
\text { invasion }\end{array}$ & $\begin{array}{c}\text { Non- } \\
\text { transmural } \\
\text { pericardial } \\
\text { invasion }\end{array}$ & $\begin{array}{c}\text { Tracheal or } \\
\text { esophageal } \\
\text { encasement }\end{array}$ & no \\
\hline Count & 1 & 6 & 3 & 10 \\
\hline$\%$ & $5.0 \%$ & $30.0 \%$ & $15.0 \%$ & $50.0 \%$ \\
\hline
\end{tabular}

- Mediastinal nodal involvement:

In the form of ipsilateral bronchopulmonary /hilar lymph nodes (N1), ipsilateral mediastinal (N2) and contralateral mediastinal or supraclavicular lymph nodes on either side (N3) Table 12. 
Table (12): Mediastinal lymph nodes involvement in MPM patients.

\begin{tabular}{|c|c|c|c|c|}
\hline & \multicolumn{3}{|c|}{ Nodal metastasis } \\
\hline & $\begin{array}{c}\text { Ipsilateral hilar/bronchopulmonary } \\
\text { lymph nodes }\end{array}$ & $\begin{array}{c}\text { Ipsilateral } \\
\text { mediastinal }\end{array}$ & $\begin{array}{c}\text { Contralateral } \\
\text { mediastinal }\end{array}$ & No \\
\hline Count & 3 & 5 & 4 & 8 \\
\hline$\%$ & $15.00 \%$ & $25.00 \%$ & $20.00 \%$ & $40.00 \%$ \\
\hline
\end{tabular}

- Diaphragmatic affection: Trans diaphragmatic spread upstages the disease to T4. About 4 cases (25\%) that showed suspected CT evidence of diaphragmatic muscle infiltration, only one case showed definite trans diaphragmatic extension Table 13.

Table (13): Showing diaphragmatic affection in the studied MPM patients.

\begin{tabular}{|c|c|c|c|}
\hline & $\begin{array}{c}\text { Trans diaphragmatic } \\
\text { invasion }\end{array}$ & $\begin{array}{c}\text { Diaphragmatic affection } \\
\text { Suspected diaphragmatic } \\
\text { muscle invasion }\end{array}$ & No \\
\hline Count & 1 & 4 & 15 \\
\hline$\%$ & $5.0 \%$ & $20.0 \%$ & $75.0 \%$ \\
\hline
\end{tabular}

- Distant metastasis: Distant metastasis in MPM is infrequent. 15 (75.0\%) of cases had no distant metastasis while $5(25.0 \%)$ of cases had distant metastasis Table 14.

Table (14): Showing distant metastasis in MPM patient.

\begin{tabular}{|c|c|c|c|c|c|}
\hline & \multicolumn{5}{|c|}{ Distant metastasis } \\
& $\begin{array}{c}\text { Multiple } \\
\text { Peritoneal } \\
\text { nodules }\end{array}$ & Hepatic deposits & $\begin{array}{c}\text { Hepatic, both } \\
\text { adrenals, and } \\
\text { posterior } \\
\text { abdominal wall }\end{array}$ & $\begin{array}{c}\text { Both adrenal } \\
\text { glands }\end{array}$ & Negative \\
\hline Count & 1 & 2 & 1 & 1 & 15 \\
\hline$\%$ & $5.0 \%$ & $10.0 \%$ & $5.0 \%$ & $5.0 \%$ & $75.0 \%$ \\
\hline
\end{tabular}

- Pathological variation Table 15.

Table (15): Shows the pathological variation of malignant pleural mesothelioma in this study proving the predominance of epithelioid type.

\begin{tabular}{|c|c|c|c|}
\hline & & Pathological variant & \\
\hline & Sarcomatoid & Epithelial type & Biphasic type \\
\hline Count & 4 & 14 & 2 \\
\hline$\%$ & $20.0 \%$ & $70.0 \%$ & $10.0 \%$ \\
\hline
\end{tabular}




\section{CASES}

CASE 1

A 38 years old male patient who presented with chest pain and dyspnea.

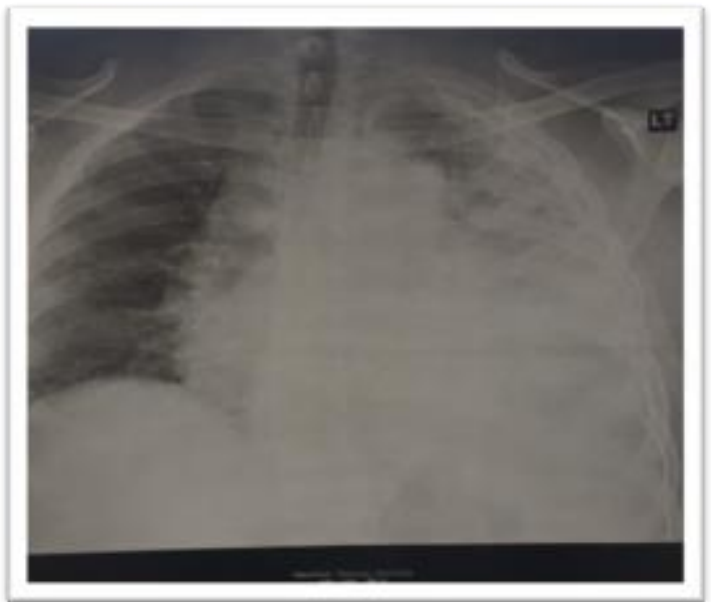

Figure1: PA chest X-Ray showing: near total left lung collapse and left pleural effusion. Also widened mediastinum??Lymphadenopathy

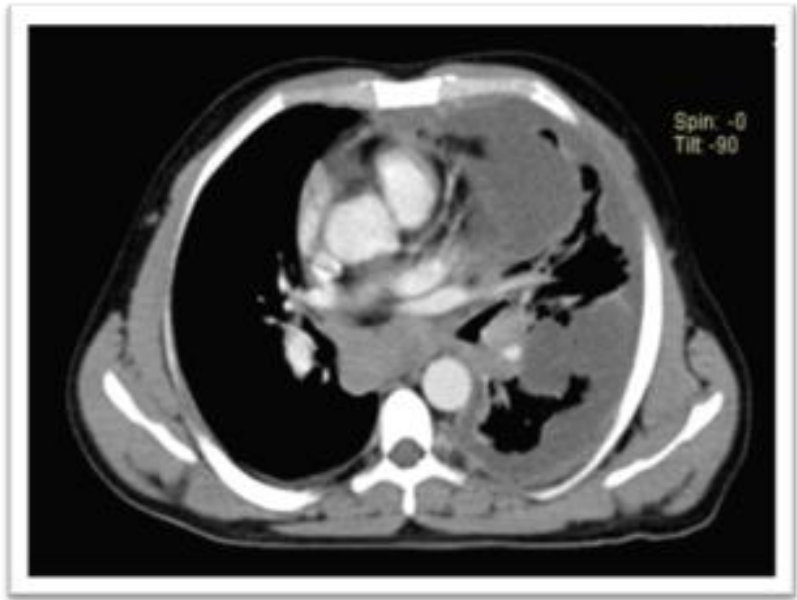

Figure 2: Axial contrast enhanced CT showing Lt sided circumferential pleural thickening involving all pleural surfaces with fissural extension and abutting the aorta.

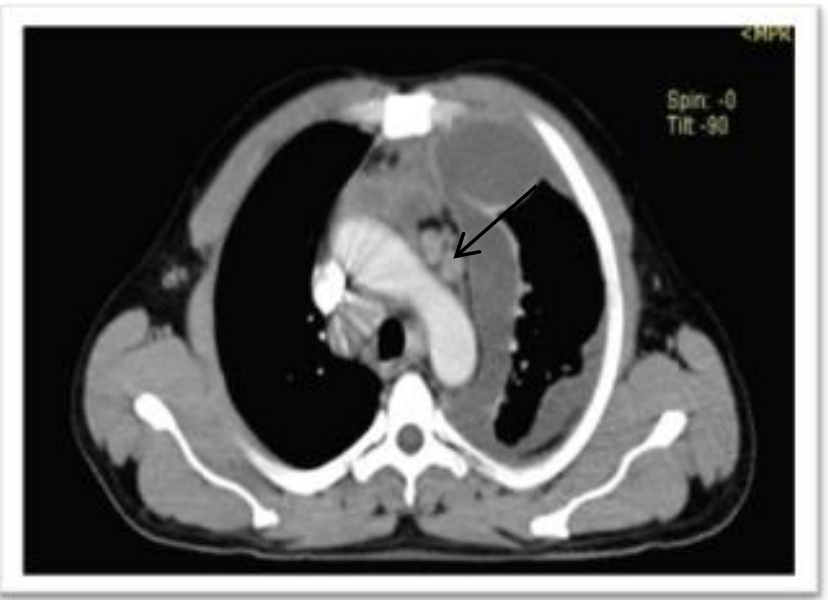

Figure 3: Axial CT scan of the chest with contrast showing: perivascular and retro-caval lymphadenopathy and pleural effusion. 
CASE 2

A80 years old male patient presenting with dyspnea and weight loss.

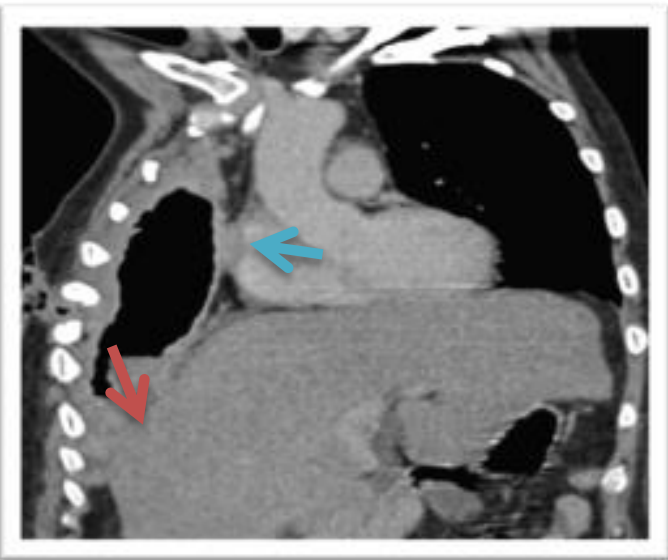

Figure 4: Coronal reformatted image of the chest showing: indistinct diaphragm with suspected diaphragmatic muscle extension and pericardial thickening (arrows).

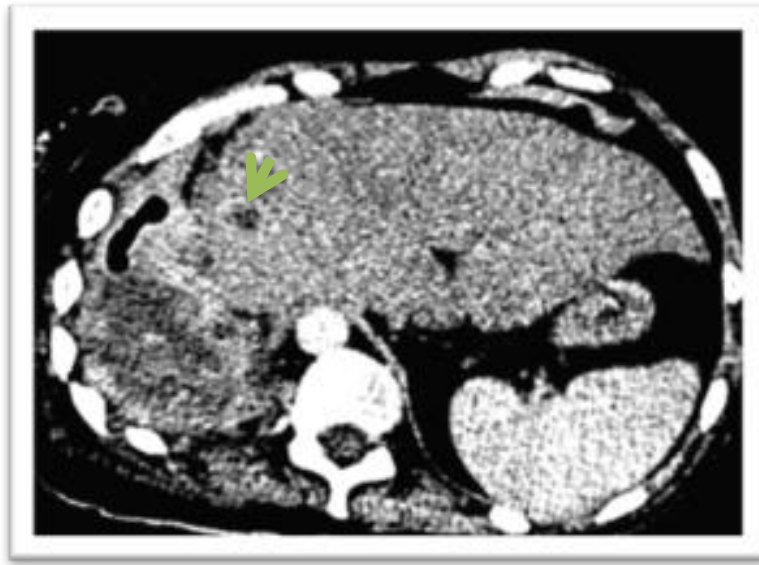

figure 5: Axial contrast enhanced CT of upper abdomen showing: hypodense marginally enhanced hepatic focal lesion.

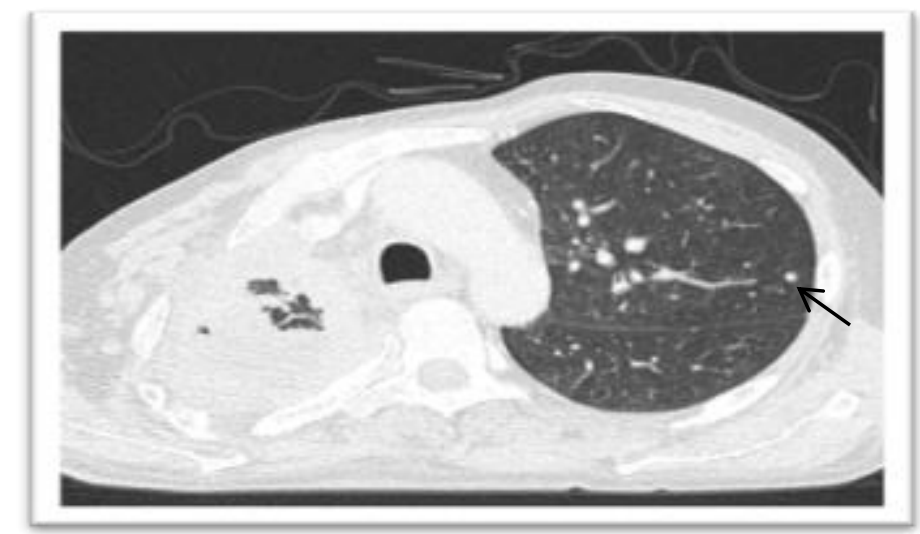

Figure 6: Axial CT chest (lung window) showing solitary left pulmonary nodule (arrow). 


\section{CASE 3}

A 49 years old male patient presenting with chest pain.

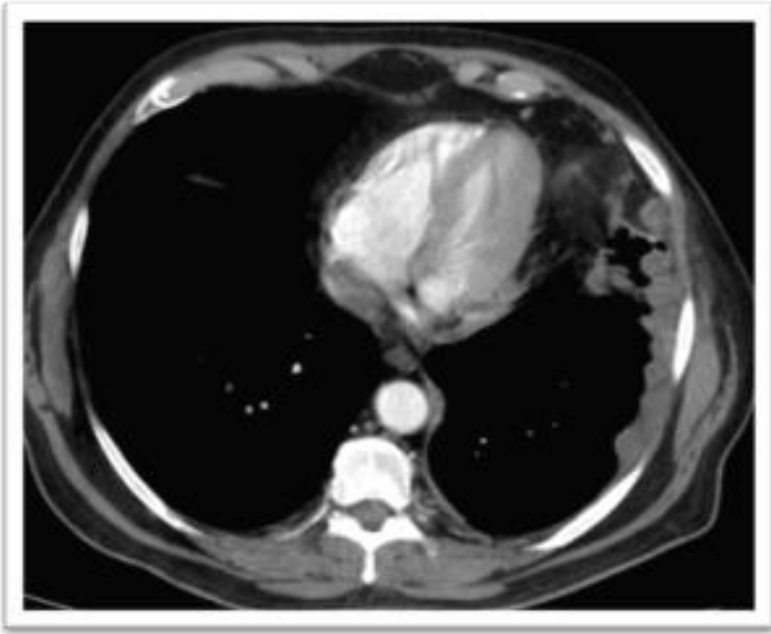

Figure 7: Axial contrast enhanced CT scan of the chest showing costal non uniform nodular left pleural thickening with nodular thickening of the left diaphragmatic crus.

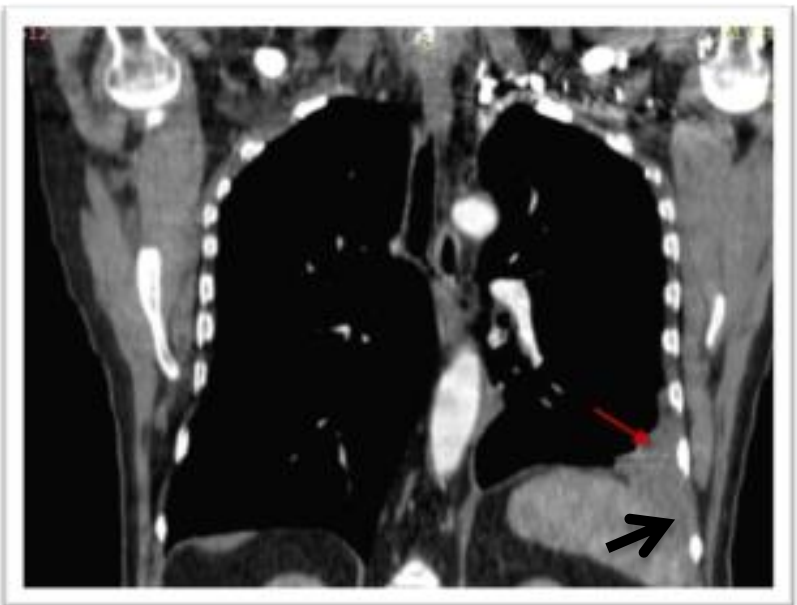

Figure 8: Coronal reformatted image of the chest showing obliteration of the left costo-phrenic recess (red arrow) and indistinct diaphragm suspecting involvement of the diaphragmatic muscle (black arrow). 


\section{DISSCUSION}

Malignant pleural mesothelioma (MPM), the commonest pleural malignancy, is a rare malignant disease yet recent studies have proven its increased incidence worldwide, ${ }^{(\boldsymbol{8})}$.

MPM is known for its aggressive nature regarding local disease extent, local spread and distant metastases. Its survival lies somewhere between 12-18 months even with treatment since it has shown resistance to different treatment options ${ }^{(4)}$.

All the latter lead to the importance of early detection and diagnosis of MPM with proper staging in order to give the patient a better chance at early treatment and a better survival time ${ }^{(5)}$. CT is the most widely used investigation for diagnosing MPM. Other modalities can be used as well like MRI and PET CT ${ }^{(2)}$.

This study showed prevalence of the disease between males which agrees with Wolf $\boldsymbol{e t}$ al. ${ }^{\left({ }^{(9)}\right.}$ who state that MPM has a higher incidence in men than women and also with Nickel et al. ${ }^{(2)}$ and Truong et al. ${ }^{(4)}$ who state that it is more common in men than in women, with a ratio of 4:1.

This study also showed that the most prevalence of the disease is in the $6^{\text {th }}$ and $7^{\text {th }}$ decades.

Agreeing with Truong et al. ${ }^{(4)}$ that MPM has a peak incidence in the sixth and seventh decades of life and Nickel $\boldsymbol{e t} \boldsymbol{a l}$. (2) that MPM most commonly occurs in patients aged $50-70$ years. This study showed the prevalence of the epitheliod variant of the three subtypes of malignant pleural mesothelioma.

The epithelioid variant was diagnosed in 14 cases representing $70 \%$ of cases. According to Anttila and Boffetta (10), epithelioid and biphasic are the most common subtypes of malignant pleural mesothelioma where together they constitute approximately $70-90 \%$ of all MPM and also describes the importance to differentiate the different subtypes in the pathology report of MPM since epithelioid MPM is known to have the best prognosis and it helps in leading the oncologist to the right treatment guidelines.

This also agrees with Nickel $\boldsymbol{e t}$ al. ${ }^{(2)}$ that epithelioid MPM is the most common type, representing $55 \%-65 \%$ of cases. This also agrees with Inai ${ }^{(11)}$ who states that the proportion of each is approximately $60 \%$ for epitheliod variant, $20 \%$ for the biphasic variant and 20\% for the sarcomatoid variant and with Allen (5) who confirms that the epitheliod variant is the most common histologic variant as well as.
$50 \%$ of the cases in this study showed right sided disease which disagrees with Allen ${ }^{(5)}$ who states that MPM is more common on the right than on the left side, in a ratio of 3:2 and with Nickel $\boldsymbol{e t}$ al. ${ }^{(2)}$ who state that the right hemi thorax is often more involved than the left.

26 cases $(65 \%)$ presented with circumferential nodular irregular pleural thickening this agrees with Cardinale et al. ${ }^{(12)}$ explain that CT features highly suggestive of the MPM include nodular or lobular circumferential pleural thickening with mediastinal pleural thickening seen in 90\%-92\% of patients.

According to Nickell et al. ${ }^{\text {(2) }}, 92 \%$ of cases show lobular/nodular pleural thickening.

Fissural involvement in this study was evident in 13 patients $(65 \%)$ which agrees with Allen ${ }^{(5)}$ who states that involvement of the inter lobar fissural pleura is characteristic of mesothelioma which can be sometimes more apparent on reformatted sagittal or coronal views compared to the conventional axial views.

Cardinale et al. ${ }^{(12)}$ also demonstrated the high incident of fissural involvement and explain that the next most common feature found in MPM after the circumferential pleural thickening is the involvement of the fissures which is seen in 73$86 \%$ of cases.

Bagheri et al. ${ }^{(13)}$ showed that the involvement of the inter lobar fissures is an important differentiating finding since it occurs in $40-86 \%$ of patients with mesothelioma.

In a recent study by Karam $\boldsymbol{e t}$ al. ${ }^{(14)}$ comparing MPM with metastatic disease, thickening of the inter lobar fissures involvement was seen in $47.1 \%$ of cases diagnosed as MPM making it an important diagnostic clue to the disease.

Dogan et al. ${ }^{(15)}$ showed that only $12 \%$ of cases had lung parenchymal involvement disagreeing with this study that showed a high percentage of lung involvement in the form of direct invasion $(60 \%)$ or parenchymal metastatic nodules $(30 \%)$ and lymphangitic spread (10\%).

Nickel et al. (2) whom say that invasion of endothoracic fascia or a single chest wall focus is better assessed by MRI and that chest wall involvement wither at sites of previous biopsy, thoracotomy, or chest tube tracts are also relatively more easily seen on MRI than on CT.

This study was able to stage the different nodal groups in the currently used staging system of MPM with $75 \%$ of cases showing affection of the ipsilateral mediastinal lymph nodes, yet Wang et al. (16) clearly states that the CT accuracy is suboptimal in proving nodal involvement 
because size only does not prove nodal involvement and that PET CT has increased accuracy when it comes to detecting mediastinal lymph nodes involvement.

Wang et al. ${ }^{(16)}$ state that trans diaphragmatic extension of MPM is suggested in CT by a softtissue mass that encases the hemi diaphragm and once a clear fat plane between the diaphragm and adjacent abdominal organs with a smooth diaphragmatic contour is seen by CT, this indicates that the tumor is limited to the thorax. This also agrees with Ismail-Khan $\boldsymbol{e t}$ al. ${ }^{\left({ }^{(17)}\right.}$ that trans diaphragmatic spread of tumor may be visible or suspected on chest $\mathrm{CT}$ scans yet MRI of the chest is more sensitive in illustrating this.

\section{CONCLUSION}

CT with contrast is the most frequently obtained examination owing to its easy accessibility. Chest CT alone is often sufficient for disease staging and treatment planning.

Typical CT findings that suggest MPM include unilateral pleural effusion with nodular irregular pleural thickening which can be discrete or diffuse with or without inter lobar fissure thickening and nodal metastasis.

The most important differential diagnosis of diffuse nodular pleural thickening includes metastatic disease.

FDG PET/CT is useful to monitor the followup and assess the metabolic response to chemo and radiotherapy.

\section{REFERENCES}

1. Thanh TD, Tho NV, Lam NS et al. (2016): Simian virus 40 may be associated with developing malignant pleural mesothelioma. Oncology Letters, 11(3):2051-2056.

2. Nickell LT, Lichtenberger JP, Khorashadi L et al. (2014): Multimodality Imaging for Characterization, Classification, and Staging of Malignant Pleural Mesothelioma. Radio Graphics, 34(2): 1692-1706.

3. Zhang W, Wu X, Wu L et al. (2015): Advances in the diagnosis, treatment and prognosis of malignant pleural mesothelioma. Ann Transl Med., 3(13):182.

4. Truong MT, Viswanathan C, Godoy MB et al. (2013): Malignant pleural mesothelioma: Role of $\mathrm{CT}, \mathrm{MRI}$, and PET/CT in staging evaluation and treatment considerations. Seminars in Roentgenology, 48(4): 323-334.

5. Allen TC (2015): Approaching the diagnosis of diffuse malignant mesothelioma. https://www.springer.com/gp/book/9781493923 731

6. Fabio F, Chiara R, Sara F et al. (2018): Imaging of malignant pleural mesothelioma: it is possible a screening or early diagnosis program?-a systematic review about the use of screening programs in a population of asbestos exposed workers. J Thorac Dis., 10(2): 262-268.

7. Faizul N, Mehdi T and Rathan S (2016): Value of FDG PET/CT in the Management of Mesothelioma. Journal of Pharmacy and Pharmacology, 16: 631-638.

8. Bueno R, Stawiski EW, Goldstein LD et al. (2016): Comprehensive genomic analysis of malignant pleural mesothelioma identifies recurrent mutations, gene fusions and splicing alterations. Nat Genet., 48(4):407-16.

9. Wolff H, Vehmas T, Oksa P et al. (2015): Asbestos, asbestosis, and cancer, the Helsinki criteria for diagnosis and attribution 2014: recommendations. Scand J Work Environ Health, 41(1):5-15.

10. Anttila S, Boffetta P (2014): Occupational cancers. https://www.springer.com/gp/book/9781447128 243

11. Inai K (2008): Pathology of mesothelioma, Environmental Health and Preventive Medicine, 13(2): 60-64.

12. Cardinale L, Francesco A, Dario G et al. (2017): Diagnostic imaging and workup of malignant pleural mesothelioma. Acta Biomed., 88(2): 134-142.

13. Bagheri R, Seyed Z, Mohammad B et al. (2011): Malignant Pleural Mesothelioma: Clinicopathologic and Survival Characteristic in a Consecutive Series of 40 Patients. Ann Thorac Cardiovasc Surg., 17: 130-136.

14. Karam MB, Shirin K, Leila $M$ et al. (2016): Malignant mesothelioma versus metastatic carcinoma of the pleura: A CT challenge. Iranian Journal of Radiology, 13(1):1-6.

15. Dogan TO, Ismail S, Fikret $T$ et al. (2012): Thoracic computed tomography findings in malignant mesothelioma. Iranian journal of radiology: a quarterly journal published by the Iranian Radiological Society, 9(4):209-11.

16. Wang ZJ, Gautham PR, Michael BG et al. (2004): Malignant Pleural Mesothelioma: Evaluation with CT, MR Imaging, and PET. Radio Graphics, 24: 105-119.

Ismail-Khan R, Lary AR and Charles CW (2006): Malignant pleural mesothelioma: a comprehensive review. Cancer control: journal of the Moffitt Cancer Center, 13(4):255-26 